\title{
Early Results after Aortic Valve Replacement Using Last Generation Bioprosthetic Aortic Valve
}

\author{
Dritan Useini, MD, Markus Schlömicher, MD, Peter Haldenwang, MD, PhD, Hamid Naraghi, MD, \\ Vadim Moustafine, MD, Matthias Bechtel, MD, PhD, Justus Strauch, MD, PhD \\ Department of Cardiothoracic Surgery, Ruhr-University Hospital Bergmannsheil, Bochum, Germany
}

\section{ABSTRACT}

Introduction: Few data are available about the newest generation surgical bioprosthesis. We aimed to evaluate early clinical and hemodynamic outcomes after using the INSPIRIS RESILIA aortic valve (Edwards Lifesciences, Irvine, California, USA).

Methods: Between July 2018 and April 2021, 80 patients underwent aortic valve replacement receiving the INSPIRIS RESILIA aortic valve at our institution. Primary outcomes were the composite of early mortality, stroke, and myocardial infarction. Secondary outcomes were hemodynamic performances of the valve, paravalvular leakage, and new pacemaker implantation.

Results: The mean age of the study population was $60.6 \pm$ 8.3 years. The mean Society of Thoracic Surgery-Predicted Risk of Mortality score was $2.9 \pm 1.7 \%$. In $43.7 \%$ of the patients, concomitant surgery was performed. The in-hospital mortality, all-stroke, and myocardial infarction rates were $2.5 \%, 1.2 \%$, and $1.2 \%$, respectively. No valve was explanted and no redo was performed. The mean postoperative transprosthetic gradient at discharge was $10.2 \pm 4.1 \mathrm{~mm} \mathrm{Hg}$. There was no need for new pacemaker implantation. We registered only two cases with minimal (trace) paravalvular leakage.

Conclusion: The use of the INSPIRIS RESILIA aortic valve in a young, low-risk population is safe and associated with very good early clinical and hemodynamic outcomes.

\section{INTRODUCTION}

The advancement of the transcatheter aortic valve replacement (TAVR) in recent years has led to fundamental changes, regarding the practice of treatment of the aortic stenosis. The utility of this method has been expanded to low-risk patients [Mack 2019]. The noninvasive nature of this method has shown very good results also in patients with degenerated bioprostheses, with foregone aortic valve replacement

Received August 1, 2021; received in revised form September 15, 2021; accepted September 15, 2021.

Correspondence: Dritan Useini, MD, Department of Cardiothoracic Surgery, Rubr-University Hospital Bergmannsheil, Bürkle de la Camp Platz 1; 44789 Bochum, Germany; +49-176-417-44123, +49-234-302-3026; fax: +49234-302-6010 (e-mail: dritan-83@live.com).
(AVR) as a valve-in-valve procedure. Even with better outcomes as the surgical redo procedures [Malik 2020]. This evolvement is changing increasingly the paradigm of the use of bioprosthetic valves over mechanical prostheses also in younger patients-supported by the American Heart Association/American College of Cardiology guidelines on valvular heart disease 2017. It lowers the threshold of recommending mechanical over bioprosthetic valves to 50 years, compared with 60 years in 2014 [Nishimura 2017]. The INSPIRIS RESILIA aortic valve (Edwards Lifesciences, Irvine, California, USA) (INSPIRIS valve), representing the last development in surgical AVR, has been created with a novel RESILIA tissue preservation technology and dry storage method and has been constituted with an extendable stent, offering the best option for later valve-in-valve treatment (Figure 1). As the INSPIRIS valve is a new valve, data regarding outcomes are rare. Therefore, we aimed to evaluate early clinical and hemodynamic outcomes.

\section{METHODS}

This single-center study was conducted to evaluate the inhospital outcomes after AVR using the latest generation of surgical bioprosthesis INSPIRIS. Between July 2018 and April 2021, 69 patients with severe aortic stenosis and 11 patients with high-grade aortic regurgitation, aged between 50 to 65 years, where a mechanical valve implantation was contraindicated or refused from patients, underwent AVR receiving the INSPIRIS valve at our institution. The study population comprised all 80 consecutive low risk, young patients. Patients with aortic valve endocarditis and concomitant procedures, such as ascending replacement, aortic root replacement, coronary surgery, mitral repair/replacement, Morrow procedure were included in the
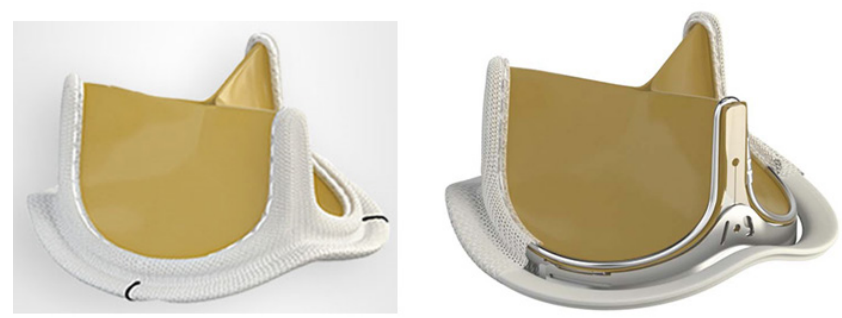

Figure 1. Edwards INSPIRIS RESILIA Aortic Valve. 
Table 1. Baseline characteristics

\begin{tabular}{|c|c|}
\hline Variable & Study group $(\mathrm{N}=80)(\%)$ \\
\hline Age (years) & $60.6 \pm 8.3$ \\
\hline Male sex & $58(72.5)$ \\
\hline Height $(\mathrm{cm})$ & $172.9 \pm 12.6$ \\
\hline Weight (kg) & $88.6 \pm 22.7$ \\
\hline STS-SCORE (\%) & $2.9 \pm 1.7$ \\
\hline Euroscore II (\%) & $3.6 \pm 2.4$ \\
\hline New York Heart Association class I/II & $40(50)$ \\
\hline New York Heart Association class III/IV & $40(50)$ \\
\hline Endocarditis & $5(6.2)$ \\
\hline Previous cardiac surgery & $7(8.7)$ \\
\hline Previous aortic valve replacement & $5(6.2)$ \\
\hline Previous coronary artery bypass & $2(2.5)$ \\
\hline Syncope & $3(3.7)$ \\
\hline Angina pectoris & $11(13.7)$ \\
\hline Hypertension & $45(56.2)$ \\
\hline Pulmonary hypertension & $3(3.7)$ \\
\hline Diabetes & $10(12.5)$ \\
\hline Coronary artery disease & $27(33.7)$ \\
\hline PTCA/PCI & $12(15)$ \\
\hline Left ventricular ejection fraction (\%) & $54.9 \pm 11.1$ \\
\hline Peripheral artery disease & $2(2.5)$ \\
\hline Previous stroke & $12(15)$ \\
\hline Chronic kidney disease & $7(8.7)$ \\
\hline Chronic obstructive pulmonary disease & $11(13.7)$ \\
\hline Atrial fibrillation & $10(12.5)$ \\
\hline
\end{tabular}

STS: Society of Thoracic Surgery-Predicted Risk of Mortality score; PTCA/ PCl: percutaneous transluminal coronary angioplasty/intervention

study. Clinical endpoints were defined according to Valve Academic Research Consortium-2 criteria [Kappetein 2013]. An echocardiographic assessment of the valve was performed at discharge. The echocardiography data at discharge were $100 \%$ available. All patients gave informed consent for data collection. Approval for the study was obtained from the Ethics Committee of the Medical Faculty of the RUHR University, Bochum, Germany (Reg. No. 19-6804).

Primary outcome was a composite of early mortality, stroke, and myocardial infarction. Secondary outcomes were hemodynamic performances of the valve, paravalvular leakage (PVL), and new pacemaker implantation (PPI) at discharge.

Distributions of quantitative variables are described as means ( \pm standard deviation). Qualitative variables are summarized by count and percentage. Comparisons of the data were assessed using student's t-test. Statistical tests were
Table 2. Procedural characteristics

\begin{tabular}{lc}
\hline & Study group \\
Variables & $(\mathrm{N}=80)(\%)$ \\
\hline Baseline $\Delta \mathrm{P}$ max (mm Hg) & $78.6 \pm 22.8$ \\
Baseline $\Delta \mathrm{P}$ mean (mm Hg) & $46.7 \pm 14.8$ \\
Aortic valve replacement & $80(100)$ \\
Contraindications of mechanical valve & \\
Well-informed patient's desire, refusing lifelong & $72(90)$ \\
anticoagulation & $3(3.7)$ \\
Women contemplating pregnancy & $2(2.5)$ \\
Redo due to mechanical valve thrombosis & $1(1.2)$ \\
Low patient compliance due to heroin-abuse & $1(1.2)$ \\
Low patient compliance due to mental retardation & $1(1.2)$ \\
Patient with cerebral cavernoma & $49(61.2)$ \\
Partial J-sternotomy/right anterior thoracotomy & $35(43.7)$ \\
Concomitant & $12(15)$ \\
Coronary artery bypass & $15(1.2)$ \\
Mitral valve replacement (Medtronic Hancook II & $26(32.5)$ \\
porcine heart valve) & $23(28.7)$ \\
Mitral valve repair & $16(20)$ \\
Replacement of ascending aorta/aortic root & $14(17.5)$ \\
Aortic root enlargement & $1(1.2)$ \\
Closure of ventricular septal defect & $1(1.2)$ \\
Morrow procedure & $24.5 \pm 18.9$ \\
Cross-clamp time (min) & $64.2 \pm 16.8$ \\
\hline
\end{tabular}

$\mathrm{CPB}$, cardiopulmonary bypass

two-sided, and $P$-values of .05 or less were considered statistically significant.

\section{RESULTS}

\section{Baseline characteristics}

Table 1 shows the preoperative data of our patient population. The mean age was $60.6 \pm 8.3$ years. The mean Society of Thoracic Surgery-Predicted Risk of Mortality score was 2.9 $\pm 1.7 \%$. Of the patients, $72.5 \%$ were male. New York Heart Association class III/IV was registered in $50 \%$ of the patients. There were five patients (6.2\%) who underwent AVR due to endocarditis. Previous aortic valve replacement was registered 


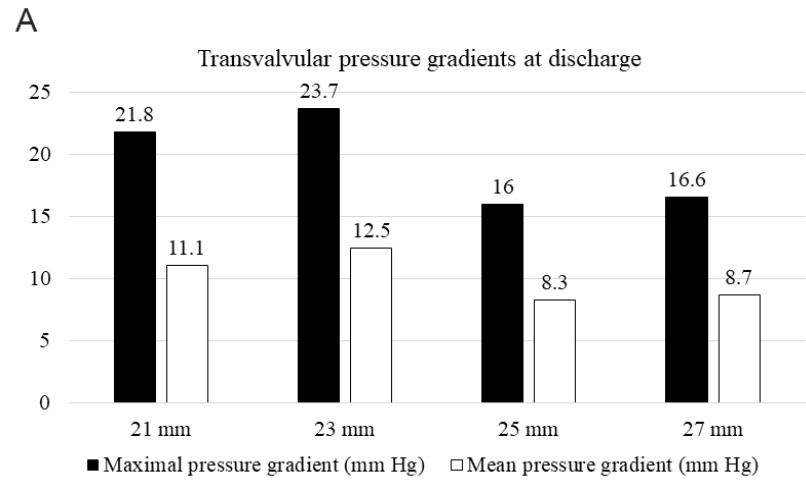

Figure 2A. Maximal and mean transvalvular pressure gradients at discharge.

in five patients $(6.2 \%)$. Previous coronary artery bypass grafting was registered in two patients $(2.5 \%)$.

\section{Procedural characteristics}

All valves successfully were implanted. There was no intra-procedural death. Vascular injury was not registered. No redo or valve-in-valve procedure were necessary. We performed concomitant procedures in 35 patients $(43.7 \%)$. Four patients (5\%) underwent mitral valve repair with ring, due to mitral annulus dilatation, and one patient $(1.2 \%)$ underwent mitral valve replacement, due to mitral valve stenosis using the Medtronic Hancook II porcine heart valve. High grade aortic regurgitation was registered in 11 patients (13.7\%). A high-grade aortic regurgitation was registered mainly due to endocarditis. Table 2 shows detailed procedural data.

\section{Postoperative anticoagulation}

Basically, our anticoagulation preference for biological valves still is the oral anticoagulation with vitamin $K$ antagonist during the first three postoperative months. In this study group, all patients underwent vitamin $\mathrm{K}$ antagonist therapy postoperatively. In patients with concomitant coronary artery disease, $100 \mathrm{mg}$ of Aspirin was given. In patients with chronic atrial fibrillation, monotherapy with vitamin $\mathrm{K}$ antagonist was the therapy regime. After the third postoperative month, a monotherapy with new oral anticoagulants was recommended.

\section{In-hospital clinical outcomes}

The in-hospital all-cause mortality and all-stroke were $2.5 \%$ and $1.2 \%$, respectively. We registered one myocardial infarction. The acute kidney injury network 2/3 (AKIN 2/3) was very low. We saw two patients with gastrointestinal complications, requiring endoscopic and surgical interventions. The new permanent pacemaker implantation rate (PPI) was $0 \%$, and the new onset left bundle branch block (LBBB) was observed only in four patients $(5 \%)$ (Table 3$)$.
B

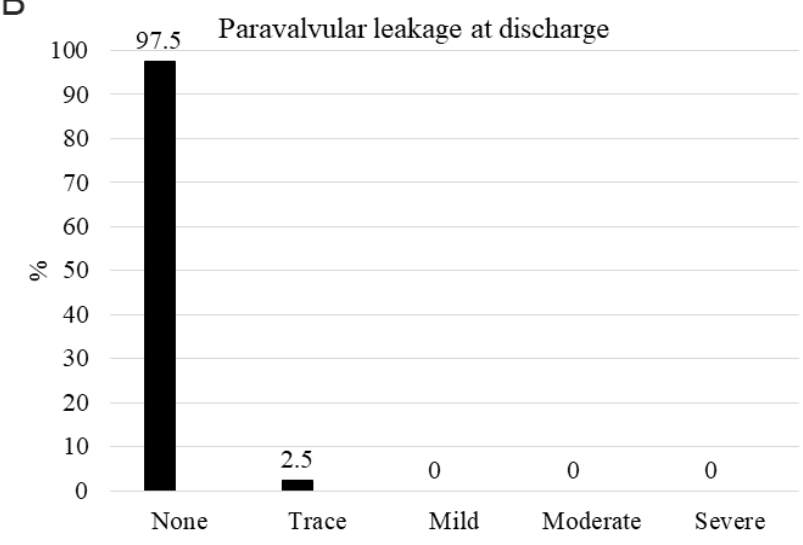

Figure 2B. Paravalvular leakage at discharge.

\section{Hemodynamics}

The baseline echocardiographic data in this study population confirmed severe aortic stenosis (Table 2). Preoperative and at discharge, the peak/mean gradients were $78.6 \pm 22.8$ / $46.7 \pm 14.8 \mathrm{mmHg}$ and $19 \pm 7.2 / 10.2 \pm 4.1 \mathrm{mmHg}$, respectively, $P<.001$. We found no severe, moderate, or mild PVL at discharge. We observed only two patients $(2.5 \%)$ with trace PVL. Of note, trace PVLs were registered in patients with bicuspid valves and after redo with INSPIRIS in previously bicuspid valve. Figures $2 \mathrm{~A}$ and $2 \mathrm{~B}$ show hemodynamic details.

\section{DIsCussION}

A mechanical valve is the first choice at our center for patients aged between 50-65 years. However, we are witnesses of a grown patient's interest toward minimally invasive procedures and better life quality in contemporary times, associated with increased request to avoid lifetime oral anticoagulation. The advancements of TAVR procedures, patient's information possibilities, and the initial education through physicians have played a crucial role in this development. As the TAVR treatment is not recommended by recent guidelines for this young population, we offer a surgical treatment with the INSPIRIS bioprosthesis, which is the most recent development in AVR technology. It represents diverse novelties in the bioprosthesis valves: The bovine pericardial tissue undergoes RESILIA tissue technology, incorporating a stable capping anticalcification process, which permanently blocks residual aldehyde groups that are known to bind with calcium. Further, tissue preservation with glycerol replaces the traditional storage in liquid-based solutions, such as glutaraldehyde. The dry storage method eliminates tissue exposure to the residual unbound aldehyde groups commonly found in glutaraldehyde storage solutions and maintains long-term protection of collagen. In contrast to the Carpentier-Edwards PERIMOUNT Magna Ease aortic bioprosthesis, in the INSPIRIS valve, the ends of the cobalt-chromium alloy band are secured by a polyester shrink sleeve on the sizes $19-25$ 
Table 3. In-hospital clinical outcomes

\begin{tabular}{lc}
\hline Variables & Study group $(\mathrm{N}=80)(\%)$ \\
\hline Hospital stay & $7 \pm 2$ \\
All-cause mortality & $2(2.5)$ \\
All stroke & $1(1.2)$ \\
Myocardial infarction & $1(1.2)$ \\
Acute kidney injury network & \\
Stage 1 & $3(3.7)$ \\
Stage 2 & $0(0)$ \\
Stage 3 & $3(3.7)$ \\
Delirium & $9(11.2)$
\end{tabular}

$\mathrm{mm}$ to allow the internal orifice of the valve to expand. The polyester support band allows expansion at each commissure when subjected to radial forces. These features are promising long durability and facility in TAVR therapies as a valve-invalve procedure in the future.

This valve has been newly introduced, and the information is minor. The key findings of the study are: 1) The use of the novel aortic bioprosthetic is safe without valve related complications. 2) The INSPIRIS valve shows very promising hemodynamics at discharge. 3) We registered no rhythm disorders requiring PPI. No mild/moderate/severe PVL were observed.

The finding of necessity of concomitant procedures in our study, representing a young and low-risk population, appears interesting. The use of the INSPIRIS valve in isolated AVR was safe and successful. The safety was reached when concomitant procedures were performed, such as mitral or ascending aorta procedures. We also used this valve in patients with endocarditis. We registered no endocarditis, valve thrombosis, or valve functional disorder. Thrombocytopenia was observed in only one patient. One patient experienced stroke, and one patient suffered myocardial infarction. Currently, very few data are reported on INSPIRIS safety. Our clinical findings are in the line with recently published safety studies [Puskas 2017; Bartuś 2018; Bartuś 2021].

It's relevant to emphasize the excellent outcomes, regarding rhythm disturbances after implantation of INSPIRIS. We registered no PPI, whereas the LBBB rate was very low. On the other side, we registered no mild, moderate, or severe PVL, and the procedures were conducted without vascular injury. It has been proven that all of these features affect the outcomes after TAVR and still represent a rigorous barrier to use TAVR in a young population.

The INSPIRIS valve was developed to enhance the durability of the bioprostheses. One of the main mechanisms to achieve the intended goal is that the RESILIA pericardial tissue undergoes an aldehyde capping process that permanently reduces calcium binding [Shang 2017]. Data up to fiveyears durability of INSPIRIS are promising [Bartuś 2021]. However, long-term data (over 15 years) are necessary to confirm the desired durability. Beyond the preservation methods and manufacturing details of the RESILIA pericardial tissue,
Table 4. Periprocedural complications

\begin{tabular}{lc}
\hline Variables & Study group $(\mathrm{N}=80)(\%)$ \\
\hline Re-thoracotomy for bleeding & $3(3.7)$ \\
Major/minor vascular complications & $0(0)$ \\
New permanent pacemaker implantation & $0(0)$ \\
Paravalvular leakage $\geq 2$ & $0(0)$ \\
$P$ mean > 20 mm Hg & $2(2.5)$ \\
Atrial fibrillation & $19(23.7)$ \\
LBBB & $4(5)$ \\
Thrombocytopenia & $1(1.2)$ \\
Valve leaflet thrombosis & $0(0)$ \\
Valve dysfunction/deterioration & $0(0)$ \\
Re-SAVR & $0(0)$ \\
Endocarditis & $0(0)$ \\
Gastrointestinal complications & $2(2.5)$ \\
Mediastinitis & $0(0)$ \\
Wound healing disorder & $0(0)$ \\
\hline
\end{tabular}

SAVR, surgical aortic valve replacement; LBBB, left bundle brunch block

relevant early outcomes, such as low transvalvular pressure gradients, absence of PVL and valve leaflet thrombosis, are paramount for long-term durability. In our study, we saw that INSPIRIS exhibited very good transvalvular pressure gradients even in the small valves. We registered only two trace PVL and no valve leaflet thrombosis. These might be early signs of a promising concept. However, long-term durability data are missing. It is well known that biological valves degenerate earlier in young patients. Renal failure is also a well-documented predictor of earlier degeneration of biovalves. Young age, in combination with renal failure, will be the most challenging population with regard to long-term durability of the INSPIRIS valve. Whether the stable capping anticalcification process of RESILIA tissue technology will overcome an early valve degeneration process in patients with renal failure with defective calcium metabolism will be a very interesting topic in the future. Actually, there are no data highlighting this important issue. Additionally, the fact that renal failure and preoperative chronic kidney disease rates are relatively low in this patient population are positive insights of our study. We registered only $3.7 \%$ stage 1 and $3.7 \%$ stage 3 acute kidney injury postoperatively, whereas the rate of the preoperative chronic kidney disease was relatively low $(8.7 \%)$.

The treatment of the aortic valve disease has reached nowadays high levels of improvement. Characterization of each available aortic valve prosthesis is of high relevance in contemporary circumstances, not only because of different methods available (TAVR, surgical AVR) with multi-approach modality (trans-femoral, trans-subclavian, trans-carotid, transapical transcatheter approaches, or minimal invasive surgical approaches, such as upper partial sternotomy or right anterior 
thoracotomy) but because of numerous different surgical and transcatheter bioprostheses, as well. The need to know which bioprosthetic valve expresses the best hemodynamic characteristics is becoming more relevant. A recent trial recently compared hemodynamic performances of a transcatheter valve with a group of surgical bioprostheses [Pibarot 2020]. However, better information will be achieved comparing each valve separately. Comparative studies with INSPIRIS valves are lacking. In a recent publication, Shala and Niclauss reported INSPIRIS to exhibit significantly better early hemodynamic characteristics than PERIMOUNT Magna Ease [Shala 2020].

The fact that INSPIRIS valve is safe with very good hemodynamic characteristics without valve-related complications, such as PPI and PVL, in accordance with the possibility to facilitate transcatheter redo interventions in the future encourages us to implant this valve in young patients who refuse to receive mechanical valves. One limitation of this study is that it is a non-randomized, single-center study with a limited number of patients.

\section{CONCLUSION}

The INSPIRIS valve can be safely implanted with very good hemodynamic performance, without early valve-related complications in young, low-risk patients.

\section{REFERENCES}

Bartuś K, Litwinowicz R, Bilewska A, et al. 2021. Final 5-year outcomes following aortic valve replacement with a RESILIA ${ }^{\mathrm{TM}}$ tissue bioprosthesis. Eur J Cardiothorac Surg. Jan 29;59(2):434-441.

Bartuś K, Litwinowicz R, Kuśmierczyk M, et al. 2018. Primary safety and effectiveness feasibility study after surgical aortic valve replacement with a new generation bioprosthesis: one-year outcomes.Kardiol Pol. 76(3):618-624.

Kappetein AP, Head SJ, Généreux P, et al. 2013. Updated standardized endpoint definitions for transcatheter aortic valve implantation: The Valve Academic Research Consortium-2 consensus document. J Thorac Cardiovasc Surg. Jan;145(1):6-23.

Mack MJ, Leon MB, Thourani, et al. 2019. PARTNER 3 Investigators. Transcatheter Aortic-Valve Replacement with a Balloon-Expandable Valve in Low-Risk Patients. N Engl J Med. May 2;380(18):1695-1705.

Malik AH, Yandrapalli S, Zaid S, et al. 2020. Valve-in-Valve Transcatheter Implantation Versus Redo Surgical Aortic Valve Replacement. Am J Cardiol. Feb 8. pii:S0002-9149(20)30115-6.

Nishimura RA, Otto CM, Bonow RO, et al. 2017. 2017 AHA/ACC focused update of the 2014 AHA/ACC guideline for the management of patients with valvular heart disease: a report of the American College of Cardiology/American Heart Association Task Force on clinical practice guidelines. Circulation. 135(25):e1159-95.

Pibarot P, Salaun E, Dahou A, et al. 2020. PARTNER 3 Investigators. Echocardiographic Results of Transcatheter Versus Surgical Aortic Valve Replacement in Low-Risk Patients: The PARTNER 3 Trial. Circulation. May 12;141(19):1527-1537.

Puskas JD, Bavaria JE, Svensson LG, et al. 2017. COMMENCE Trial Investigators.

The COMMENCE trial: 2-year outcomes with an aortic bioprosthesis with RESILIA tissue.

Eur J Cardiothorac Surg. Sep 1;52(3):432-439.

Shala M and Niclauss L. 2020. Early results of the Resilia Inspiris aortic valve in the old age patients - a retrospective comparison with the Carpentier Edwards Magna Ease.J Cardiovasc Thorac Res. 12(3):222-226.

Shang H, Claessens SM, Tian B, Wright GA. 2017. Aldehyde reduction in a novel pericardial tissue reduces calcification using rabbit intramuscular model. J Mater Sci Mater Med. 28:16. 